\title{
Familial hemophagocytic lymphohistiocytosis
}

INSERM

\section{Source}

INSERM. (1999). Orphanet: an online rare disease and orphan drug data base. Familial hemophagocytic lymphohistiocytosis. ORPHA:540

Familial Hemophagocytic lymphohistiocytosis (FHL) is a rare primary immunodeficiency characterized by a macrophage activation syndrome (see this term) with an onset usually occurring within a few months or less common several years after birth. 